\title{
Comparison of Histopathology of Transverse Carpal Ligament in Patients with Idiopathic Carpal Tunnel Syndrome and Hemodialysis Patients with Carpal Tunnel Syndrome
}

\author{
Erdinc Civelek ${ }^{1}$, Tufan Cansever ${ }^{1}$, Serdar Kabatas ${ }^{1}$, Ebru Demiralay ${ }^{2}$, \\ Emre Demircay ${ }^{3}$, Cem Comunoglu ${ }^{2}$, Cem Yilmaz ${ }^{4}$, Nur Altınors ${ }^{4}$ \\ ${ }^{1}$ Department of Neurosurgery, Baskent University Istanbul Hospital, Istanbul, Turkey \\ ${ }^{2}$ Department of Pathology, Baskent University Istanbul Hospital, Istanbul, Turkey \\ ${ }^{3}$ Department of Orthopedics, Baskent University Istanbul Hospital, Istanbul, Turkey \\ ${ }^{4}$ Department of Neurosurgery, Baskent University, Ankara, Turkey \\ E-mail: civsurgeon@yahoo.com
}

Received August 14, 2010; revised September 15, 2010; accepted September 25, 2010

\begin{abstract}
The aim of this paper is to point out the growing clinical importance of Carpal tunnel syndrome in patients on hemodialysis especially in aspect of the diagnosis, treatment and possible cause of the syndrome. Sixty patients with clinical diagnosis of Carpal Tunnel Syndrome was defined as the presence of two subjective symptoms (numbness, tingling in the median nerve distribution). The diagnosis was confirmed by electromyography. The patients with rheumatoid arthritis, thalasemia and thyroid dysfunction and the patients having pain due to arthritis or tenosynovitis were excluded. Fifty patients with clinical diagnosis of idiopathic CTS and seven hemodialysis patients having CTS were analyzed. Of 50 patients ( 47 female, 3 male) with clinical diagnosis of idiopathic CTS, 11 patients (22\%) were involved bilaterally, 25 patients (50\%) were affected only on the right and 14 patients $(28 \%)$ were symptomatic only on the left. Of 7 hemodialysis patients ( 2 female, 5 male) with CTS, 1 patient (14\%) was involved bilaterally (having two-sided A-V fistula), 4 patients $(57 \%)$ were affected only on the right and 2 patients $(28 \%)$ were symptomatic only on the left. There was significant correlation between the arteriovenous fistula and subsequent development of CTS. The all patients had fistulas in the affected side ( 5 of them were patent and 2 were occluded). In the relation between the duration of hemodialysis and development of CTS, 4 patients were over 10 years of hemodialysis, 2 patients were between 5 to 9 years and only 1 patient was below 4 years of duration. Amyloid deposit was demonstrated in 4 of 7 operated hands in the hemodialysis group. When we compared the presence of amyloid deposits in these groups, the difference between these two groups were found as statistically significant $(\mathrm{p}<0.009)$. Although hemodialysis has no significant effect on development of fibrosis, the incidence of fibrosis was found as statistically significant in idiopathic carpal tunnel syndrome $(\mathrm{p}<0.048)$. It is likely that there are numerous factors that may act either independently or in concert to potentiate the risk for developing CTS in patients on long-term hemodialysis.
\end{abstract}

Keywords: Carpal Tunnel Syndrome, Hemodialysis, Chronic Renal Failure

\section{Introduction}

Neurological complications in patients with renal failure and hemodialysis are well-known. Among them peripheral nerve involvement has been considered as a toxic polyneuropathy affecting mainly distal parts of the four extremities. Entrapment neuropathy such as Carpal tunnel syndrome (CTS) could be completely curable with defined diagnosis and proper treatment $[1,2]$.

CTS is divided into two large groups; idiopathic CTS and symptomatic CTS. Symptomatic CTS may range from due to traumas, anomalies, inflammation, neo- 
plasms, rheumatism, metabolic disorders and etc. Among them we list a hemodialysis as a one of crucial causes of symptomatic CTS. A raised intracarpal canal pressure results in median nerve compression and impaired nerve perfusion that leads to discomfort and paresthesias in the one or both hands. In early stages, patients usually complain of symptoms due to the involvement of the sensory component of the median nerve and only later report symptoms from involvement of motor fibers. Patients may also complain of pain radiating to the forearm, elbow or even the shoulder.

The exact pathogenesis of CTS is not clear. Several theories are present to explain the symptoms and impaired nerve conduction studies. The most popular ones are mechanical compression, micro-vascular insufficiency, and vibration theories $[3,4]$.

The patient with mild symptoms of CTS can be managed with conservative treatment, particularly local injection of steroids. However, in moderate to severe cases, surgery is the only treatment that provides cure. The basic principle of surgery is to increase the volume of the carpal tunnel by dividing transverse carpal ligament to release the pressure on the median nerve.

The aim of this paper is to point out the growing clinical importance of CTS in patients on hemodialysis especially in aspect of the diagnosis, treatment and possible cause of the syndrome.

\section{Methods}

All patients (the number of 60) in the study had a clinical diagnosis of Carpal Tunnel Syndrome (CTS), which was defined as the presence of two subjective symptoms (numbness, tingling in the median nerve distribution). Electromyography was examined in the abductor pollicis brevis, first dorsal interosseous and flexor carpi radialis muscles with concentric needle electrodes. The CTS diagnosis was confirmed by electromyography. A total of 10 patients had one or more associated predisposing factors or conditions (renal failure $=7$; rheumatoid arthritis $=1$; thalasemia $=1$; thyroid dysfunction $=1$ ). The patients with rheumatoid arthritis, thalasemia and thyroid dysfunction and the patients having pain due to arthritis or tenosynovitis were excluded. Fifty patients with clinical diagnosis of idiopathic CTS and seven hemodialysis patients having CTS were analyzed.

The median age was 65 years (range $42-83$ years), with a mean follow-up period of 14 months (range 12 to 26 months). Decompression of the carpal tunnel was performed using a standard open technique, which involved a 3-4 cm curvilinear incision. The palmar fascia and flexor retinaculum was then divided under direct vision, taking care to protect the recurrent motor branch.
Sectioned specimens of the transverse carpal ligament of operated cases were stained with Hematoxylene (H) and eosin (E) and crystal violet and examined with light and polarized-light microscopes (Figures 1,2 and 3). The specimens from transverse carpal ligament were pathologically evaluated according to parameters of presence of amyloid deposits, fibrosis, edema, lipomatosis, vascular proliferation, and perivascular lenfositic infiltration. Semi-quantatively, edema, fibrosis and vascular proliferation were classified as none (0), mild (1), moderate (2), lipomatosis, perivascular lymphocyte infiltration and amyloid deposition were classified as none( 0 ) and present (1) (Table 1). The pathological results were compared statistically by using linear regression test. $P$ value less than 0.01 and 0.05 was accepted as significant.

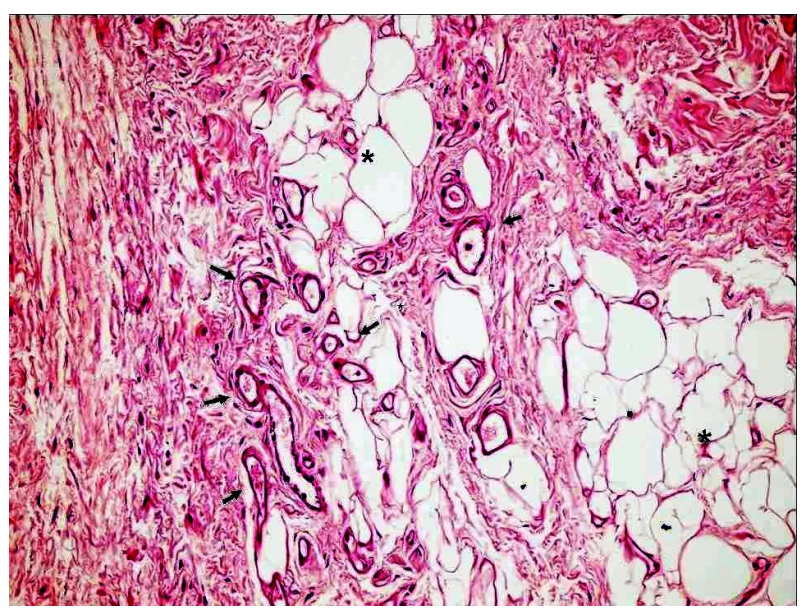

Figure 1. Hematoxylene and eosin staining of specimen of transverse carpal ligament showing vascular proliferation (arrows) and lipomatosis (asterisks) (H\&E x 100).

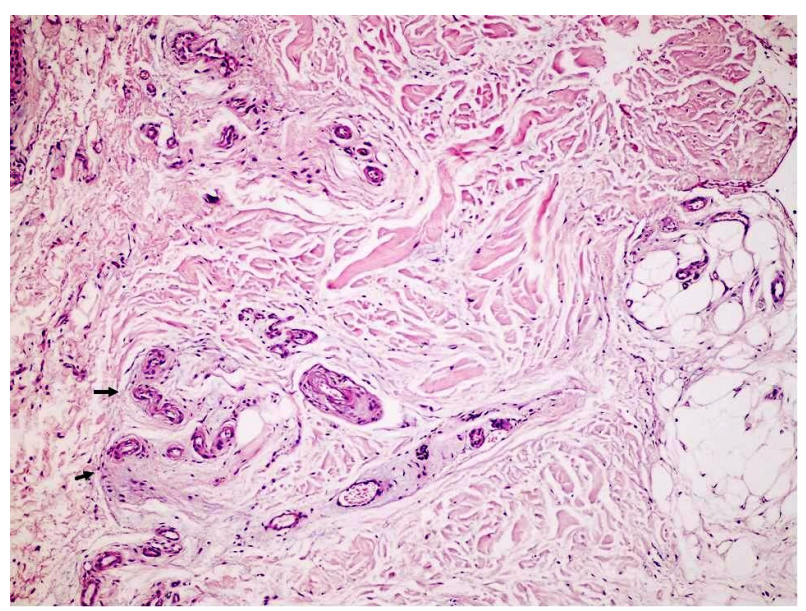

Figure 2. Hematoxylene and eosin staining of specimen of transverse carpal ligament showing perivascular lymphocyte infiltration (arrows) (H\&E x 100). 


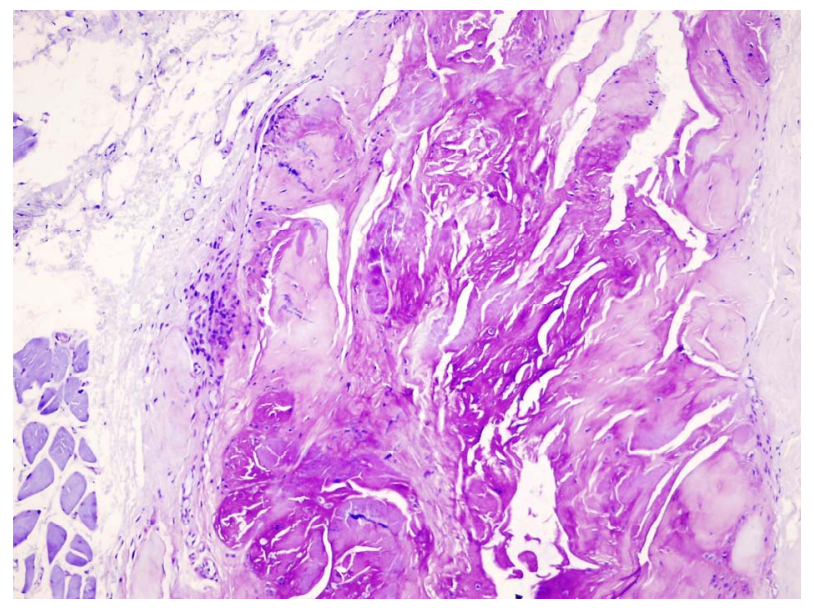

Figure 3. Crystal violet staining of specimen of transverse carpal ligament showing amyloid deposits (Crystal violet $x$ 100).

Table 1. Semi-quantities measurement criteria of edema, vascular proliferation and fibrosis.

\begin{tabular}{cccc}
\hline Grade & Edema & $\begin{array}{c}\text { Vascular prolifera- } \\
\text { tion }\end{array}$ & Fibrosis \\
\hline $\mathbf{0}$ & none & none & none \\
$\mathbf{1}$ & $\begin{array}{c}\text { Minimal dissocia- } \\
\text { tion of collagen } \\
\text { bands }\end{array}$ & $\begin{array}{c}\text { Minimal increase in } \\
\text { vascular structures }\end{array}$ & $\begin{array}{c}\text { Minimal increase } \\
\text { in number of fibro- } \\
\text { blasts }\end{array}$ \\
& & & \\
& $\begin{array}{c}\text { Marked dissocia- } \\
\text { tion of collagen } \\
\text { bands and fluid } \\
\text { between the bands }\end{array}$ & $\begin{array}{c}\text { Intense clustering of } \\
\text { vascular structures }\end{array}$ & \\
& &
\end{tabular}

\section{Findings}

Of 50 patients (47 female, 3 male) with clinical diagnosis of idiopathic CTS, 11 patients $(22 \%)$ were involved bilaterally, 25 patients $(50 \%)$ were affected only on the right and 14 patients $(28 \%)$ were symptomatic only on the left. Of 7 hemodialysis patients ( 2 female, 5 male) with CTS, 1 patient (14\%) was involved bilaterally (having two-sided A-V fistula), 4 patients (57\%) were affected only on the right and 2 patients (28\%) were symptomatic only on the left. There was significant correlation between the arteriovenous fistula and subsequent development of CTS. The all patients had fistulas in the affected side ( 5 of them were patent and 2 were occluded). In the relation between the duration of hemodialysis and development of CTS, 4 patients were over 10 years of hemodialysis, 2 patients were between 5 to 9 years and only 1 patient was below 4 years of duration.

Amyloid deposit was demonstrated in 4 of 7 operated hands in the hemodialysis group. When we compared the presence of amyloid deposits in these groups, the difference between these two groups were found as statistically significant $(\mathrm{p}<0.009)$.

Fibrosis was not observed in the hemodialysis group, but it was demonstrated in 19 of 50 operated hands in the idiopathic group. Although hemodialysis has no significant effect on development of fibrosis, the incidence of fibrosis was found as statistically significant in idiopathic carpal tunnel syndrome $(p<0.048)$. This may be due to slow progression of the pathology of the idiopathic carpal tunnel syndrome.

When we compared these two groups according to parameters of presence of edema, lipomatosis, vascular proliferation, and perivascular lenfositic infiltration, there was no significant difference (Table 2).

Paresthesia such as sensation of swelling, numbness or tingling of the first three radial digits and radial volar aspect of the palm were noted in $100 \%$ of the hands. Pain in the first radial digits and wrist were particularly severe at night and sometimes during hemodialysis. Motor weakness and muscle atrophy were found only in the thenar muscles of the advanced cases. Tinel's and Phalen's signs were demonstrated in $45 \%$ and $36 \%$ of the symptomatic hands, respectively.

No patients developed minor wound infection postoperatively. Following the operation, all symptoms were disappeared in a month except slight numbness in the small area of the second and third finger tips.

\section{Results and Discussion}

Carpal tunnel syndrome is the most common form of peripheral neuropathy, affecting around 0.1 to $1 \%$ of the

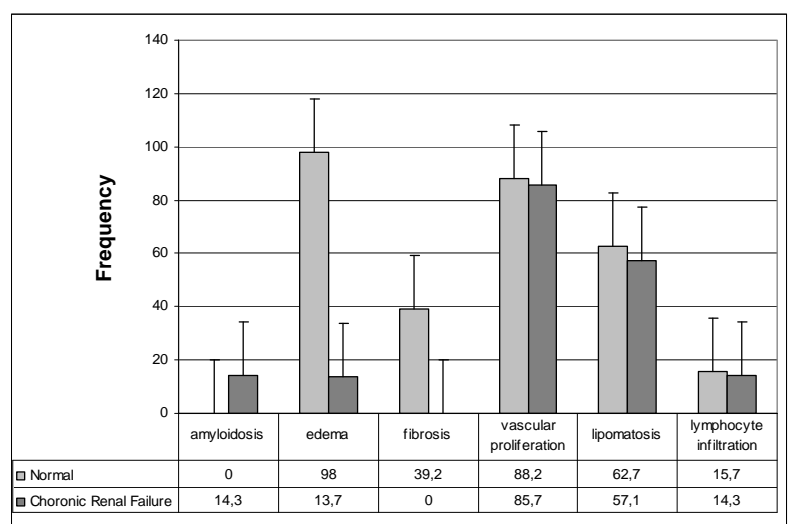

Table 2. Comparison of the hemodialysis (chronic renal failure) and the idiopathic group according to parameters of presence of amyloid deposits, fibrosis, edema, lipomatosis, vascular proliferation, and perivascular lenfositic infiltration. 
population with an estimated lifetime risk of $10 \%$. In hemodialysis patients, the incidence of Carpal tunnel syndrome is $4-15 \%$. The carpal tunnel syndrome is commonly seen in patients who have received hemodialysis for a long time $[5,6]$. The prevalence of the syndrome increases with the duration of dialysis, and nearly all patients treated for more than 18 years require a decompression operation $[5,6]$. In an other study, the percentage of patients requiring surgery was none before 8 years, $50 \%$ of those on hemodialysis for 14 years and $100 \%$ of patients on hemodialysis for 20 years [7]. In the relation between the duration of hemodialysis and development of CTS, 4 patients were over 10 years of hemodialysis, 2 patients were between 5 to 9 years and only 1 patient was below 4 years of duration in our study.

Pathogenesis of CTS in hemodialysis patients has not been fully explained. It may differ somewhat from that of idiopathic CTS. The incidence of CTS appears to be higher in hemodialysis patients than commonly estimated rate in general population. A relatively high incidence of male patients ( $71 \%$ in our series) is another characteristic point in CTS in hemodialysis patients. In idiopathic CTS, a predominance of female patients $(60-80 \%)$ has been generally described $[8,9,10]$. It was $94 \%$ in our study.

Dialysis-induced amyloidosis is a well-known complication involving deposition of amyloid fibrils that consists of $\beta 2$-microglobulin. It has been suggested that the deposition of this new form of amyloid is related to chronic immunologic stimulation during hemodialysis with the use of poorly biocompatible membranes [11]. But, the other reports have shown that $\beta 2$-microglobulin precipitates in vitro to form amyloid. Because the circulating levels of $\beta 2$-microglobulin are elevated in cases of renal failure, an accumulation of $\beta 2$-microglobulin and subsequent formation of amyloid deposits in tissue is possible $[12,13]$. In hemodialysis patients, Kachel et al. investigated other tissue sites and demonstrated amyloid deposits in the peripheral tissue, skins and rectum [2]. In the present study, it is possible to confirm the relationship between the development of CTS in hemodialysis patients and deposits of amyloid in the carpal tunnel. Miyasaka et al. detected several macrophage-like cells around local blood vessels as well as invading the synovial tissues of CTS, and discovered that these cells were positive for IL-1 and IL-6 [14].

In the literature, one possible explanation for the development of CTS during hemodialysis could be an increased pressure in the carpal tunnel due to periodic increase of total body water and venostasis [15]. The increased pressure then leads to impaired microcirculation around the carpal tunnel. This circulatory hypothesis may explain a transient relief of the symptoms following hemodialysis and an exacerbation at night. The high incidence of CTS in patients with generalized edema due to myxoedema and pregnancy may also support this hypothesis.

A close relationship between CTS and presence of an arteriovenous fistula has been postulated [15]. High rates of blood flow through the fistula may lead to nerve entrapment. High rates of blood flow through the fistula may lead to nerve entrapment. High rates of blood flow may cause an increased venous pressure and engorgement of the structures within the carpal tunnel. An alternative possibility is that high rates of blood flow may lead to ischemia within the carpal tunnel and may predispose the nerve to injury [16]. In our study, the all patients had fistulas in the affected side (5 of them were patent and 2 were occluded). Anatomic changes in the wrist area due to the fistula are probably important in the development of this syndrome.

\section{Conclusions}

It is likely that there are numerous factors that may act either independently or in concert to potentiate the risk for developing CTS in patients on long-term hemodialysis. Further investigations are necessary to substantiate the pathogenesis of CTS.

\section{References}

[1] V. K. Jain, R. V. Cestero and J. Baum, "Carpal Tunnel Syndrome in Patients Undergoing Maintenance Hemodialysis," Journal of the American Veterinary Medical Association, Vol. 242, No. 26, 1979, pp. 2868-2869. doi:10.1001/jama.242.26.2868

[2] H. G. Kachel, P. Altmeyer, C. A. Baldamus and K. Koch, "Deposition of an Amyloid-like Substance as a Possible Complication of Regular Dialysis Treatment," Contributions to Nephrology, Vol. 36, 1983, pp. 127-132.

[3] W. R. Brian and A. D. Wright, "Spontaneous Compression of Both Median Nerves in the Carpal Tunnel," Lancet, Vol. 1, 1947, pp. 277-282.

[4] E. Viikari-Juntura and B. Silverstein, "Role of Physical Load Factors in Carpal Tunnel Syndrome," Scandinavian Journal of Work, Environment \& Health, Vol. 25, No. 5, 1999, pp. 163-185.

[5] T. Bardin, J. Zingraff, D. Kuntz and T. Drueke, "Dialsis-related Amyloidosis," Nephrol Dial Transplant, Vol. 1, No. 3, 1986, pp.151-154.

[6] E. A. Brown, I. R. Arnold and P. E. Gower, "Dialysis Arthropathy: A Complication of Long-term Treatment with Haemodialysis," British Medical Journal, Vol. 292, No. 6514, 1986, pp. 163-166.

[7] Y. Shimizu, A. Van Sevevter, K. J. Horgan and S. Shaw, "Regulated Expression and Binding of Three VLA (beta 
1) Integrin Receptors on Tcells," Nature, Vol. 345, No. 6272, 1990, pp.250-253. doi:10.1038/345250a0

[8] G. S. Phalen, "Reflections on 21 Years Experience with the Carpal-tunnel Syndrome," Journal of the American Medical Association, Vol. 212, No. 8, 1970, pp.13651367.

[9] G. S. Phalen, "The Carpal-tunnel Syndrome. Clinical Evaluation of 598 Hands," Clinical Orthopaedics and Related Research, Vol. 83, 1972, pp.29-40. doi:10.1097/00003086-197203000-00007

[10] I. Kimura and D. R. Ayyar, "The Carpal Tunnel Syndrome: Electrophysiological Aspects of 639 Symptomatic Extremities," Electromyography \& Clinical Neurophysiology, Vol. 25, No. 2-3, 1985, pp. 151- 164.

[11] J. Chanard, S. Lavaud, O. Toupance, H. Roujouleh and J. P. Melin, "Carpal Tunnel Syndrome and Type of Dialysis Membrane Used in Patients Undergoing Long-term Hemodialysis," Arthritis Rheum, Vol. 29, No. 9, 1986, pp. 1170-1171. doi:10.1002/art.1780290919

[12] R. F. Gagnon, P. Somerville and M. Kaye, " $\beta 2-m i c r o-$ globulin Serum Levels in Patients on Long-term Dialy- sis," Perit Dial Bull, Vol. 7, No. 6, 1987, pp. 29-31.

[13] L. H. Connors, T. Shirahama, M. Skinner, A. Fenves and A. S. Cohen, "In Vitro Formation of Amyloid Fibrils from Intact $\beta 2$-microglobulin," Biochemical and Biophysical Research Communications, Vol. 131, No. 3, 1985, pp. 1063-1068.

doi:10.1016/0006-291X(85)90198-6

[14] N. Miyasaka, K. Sato, Y. Kitano, M. Higaki, K. Nishioka and K. Ohta, "Aberrant Cytokine Production from Tenosynovium in Dialysis Associated Amyloidosis," Annals of the Rheumatic Diseases, Vol. 51, No. 6, 1992, pp.797-802.

[15] J. A. Delmez, B. Holtmann, G. A. Sicard, A. P. Goldberg and H. R. Harter, "Peripheral Nerve Entrapment Syndromes in Chronic Hemodialysis Patients," Nephron, Vol. 30, No. 2, 1982, pp.118-123. doi:10.1159/000182447

[16] J. A. Bussell, J. A. Abbot and R. C. Lim, "A Radial Steal Syndrome with Arteriovenous Fistula for Hemodialysis. Studies in Seven Patients," Annals of Internal Medicine, Vol. 75, No. 3, 1971, pp.387-394. 